\title{
Natural conception and successful live birth in a woman after unilateral oophorectomy for ovarian cancer
}

\author{
Yue Teng \\ Department of Obstetrics and Gynecology, the First Affiliated Hospital of \\ Xi'an Jiaotong University, Xi'an 710061, China
}

\begin{abstract}
Infertility is a common complaint of woman presenting with EOC (epithelial ovarian cancer) at reproductive age. Fertility sparing treatments have been successfully attempted in ovarian cancer, while limited evidence is available to testify the postoperational fertility conservation. A 21-year-old woman (gravida 0, para 0) complaining of abdominal distension, dull pain in the lower abdomen, and vomiting was hospitalized. Unilateral oophorectomy and multiple peritoneal sampling were scheduled. Intraoperational frozen section analysis (FSA) showed "borderline mucinous ovarian tumor, accompanied with local intraepithelial carcinogenesis", indicating a pathological diagnosis of stage IA EOC. No courses of chemotherapy were given postoperatively, and regular follow-ups were scheduled every three months, with no positive results found. Eighteen months after the operation, the patient got natural conception and gave birth to a live term infant by natural delivery. Conservative surgery and close follow-ups may serve as a reasonable option for young women desiring childbearing in the treatment of early-stage EOC.
\end{abstract}

Keywords: Epithelial ovarian cancer; Fertility sparing; Conservative EOC treatment

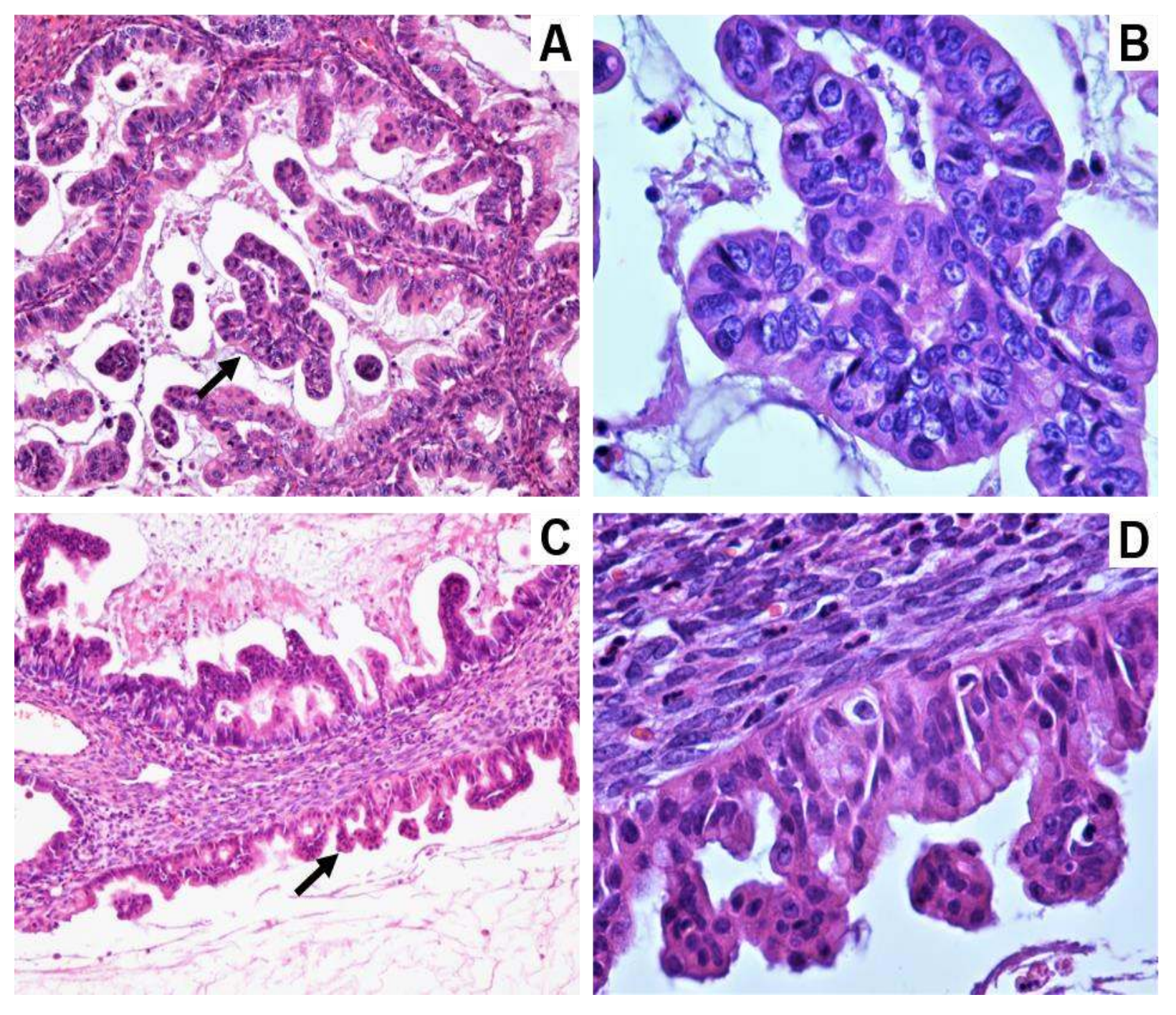

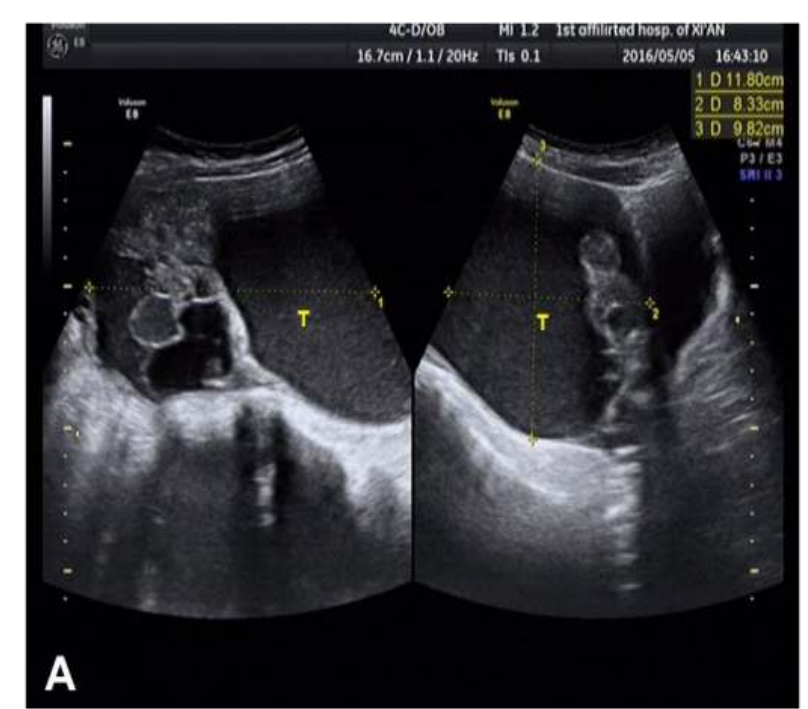

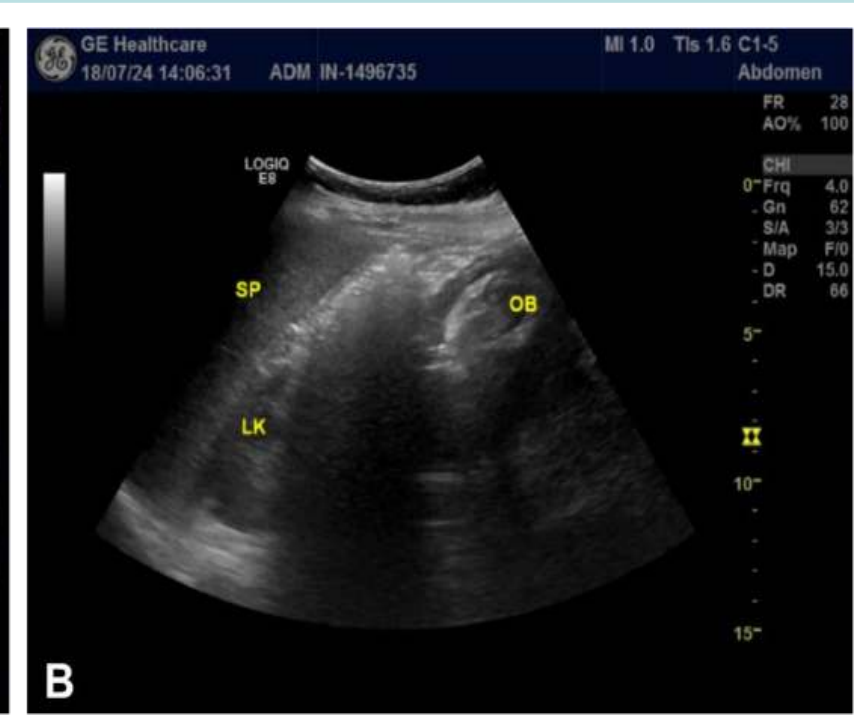

Figure 1 Histological findings. "borderline mucinous ovarian tumor $(C, D)$, accompanied with local intraepithelial carcinogenesis $(A, B) " . H \& E, \times 100(A, C) / \times 400(B, D)$.

Figure 2 Sonography findings. A: Abdominal ultrasonography at the first admission; $\mathrm{B}$ : Abdominal ultrasonography at the second admission(2 days before the delivery). Abbreviations: T: tumor; SP: spleen; LK: left kidney; OB: fetus. 